\title{
There goes the neighborhood: Assembly of transcriptional complexes during the regulation of metabolism and inflammation by the Glucocorticoid Receptor
}

Greulich, Franziska ${ }^{1,3}$; Hemmer, M. Charlotte ${ }^{1,3}$; Rollins, David A. ${ }^{2}$, Rogatsky, Inez ${ }^{2}$ and Uhlenhaut, N. Henriette ${ }^{1,4}$

1: Helmholtz Diabetes Center (HMGU) and German Center for Diabetes Research (DZD), IDO, Parkring 13, 85748 Garching; Munich, Germany

2: Hospital for Special Surgery, The David Rosensweig Genomics Center, 535 East 70th Street, New York, NY 10021, USA and Graduate Program in Immunology and Microbial Pathogenesis, Weill Cornell Graduate School of Medical Sciences, 1300 York Avenue, New York, NY 10021, USA

3: These authors contributed equally to this work

4: Corresponding author: Uhlenhaut, N.H.: henriette.uhlenhaut@helmholtzmuenchen.de 


\section{Abstract}

Glucocorticoids (GCs), as ligands for the glucocorticoid receptor (GR), represent one of the most effective and frequently used classes of drugs for anti-inflammatory and immunosuppressive therapy. In addition, its role in physiological and pathophysiological processes makes the GR an important research target. The past decades have yielded a wealth of insight into the physiological and pharmacological effects of GCs. Today's era of next generation sequencing techniques is now beginning to elucidate the molecular and genomic circuits underlying GR's cell type-specific actions. This review focuses on the concepts and insights gained from recent studies in two of the most important tissues for GC action: the liver (mediating GR's metabolic effects) and macrophages (as the main target of anti-inflammatory GC therapy). We summarize results obtained from transgenic mouse models, molecular and genome-wide studies to illustrate GR's complex interactions with DNA, chromatin, co-regulators and other transcription factors. Characterizing the cell type-specific transcriptional complexes assembled around GR will pave the road for the development of new anti-inflammatory and metabolic therapies in the future.

\section{Keywords:}

Glucocorticoid Receptor, inflammation, metabolism, transcription, genomics, coregulators 
1. Introduction

2. Who's talking? GR's neighborhood in hepatocytes and macrophages

2.1 GR acts as a master regulator of glucose metabolism in liver

2.1.1 Endowing competence for hepatocyte differentiation: Neighboring motifs in liver

2.2 GR signaling in macrophage inflammation

2.2.1 Towards a myeloid lineage: Role of priming factors in macrophages

3. GR and its coregulators

3.1 Nuclear Receptor Coactivators (NCOAs) of the p160 family

3.2 TRIP6

3.3. Hydrogen Peroxide-Inducible Clone 5 (HIC5)

4. Shaping cell-type specification

4.1 Role of the epigenetic landscape in GR gene regulation

4.2 Coregulator specificity towards certain physiological pathways

5. Future directions 


\section{Introduction}

The glucocorticoid receptor (GR) belongs to the nuclear hormone receptor family of ligand-activated transcription factors. With their widespread influence on body homeostasis, metabolism, the immune system and embryonic development, nuclear receptors (NRs) are prime targets to study the nature of physiological responses in health and disease [1]. Glucocorticoids (GCs) are a class of steroid hormones that act as ligands for GR. By the middle of the last century, the anti-inflammatory and immunosuppressive potential of the GC-GR axis has gained widespread significance and relevance as powerful therapy for inflammatory diseases [2]. In normal physiology, GCs control systemic energy homeostasis and the body's stress response through the hypothalamic-pituitary-adrenal (HPA) axis [3]. GR knockout mice do not survive beyond a few hours after birth due to respiratory failure, further demonstrating GR's important role for survival [4]. Unfortunately, its beneficial anti-inflammatory potential used in GC therapy comes along with a wide array of detrimental side effects, many of which are linked to GR's powerful role as a regulator of metabolic genes. Some of those metabolic side effects are shared with patients of Cushing's disease, which present with high circulating GC levels and associated hyperglycemia, hepatic steatosis, insulin resistance and central obesity $[5,6]$.

At the cellular level, activated GC-bound GR translocates from the cytoplasm into the nucleus where it both positively and negatively regulates the expression of genes. GR can directly bind to glucocorticoid response elements (GREs), classically defined as inverted repeats (5'nGnACAnnnTGTnCn-3') in the DNA sequence of promoters and enhancers of target genes [7, 8]. GR can influence gene expression either directly or by interaction with other DNA-bound transcription factors or via composite elements (a combination of GRE and other motifs in close proximity) [9, 10]. Ligand-activated GR does not act in isolation; rather, it recruits an array of cofactors/coregulators thereby assembling large multiprotein complexes that ultimately affect gene expression.

With the increasing amount of genomic data on hand, the past years have shown that GR targets are highly regulated in a time- (circadian rhythm, 
signals and stimuli) and space- (cell type) dependent manner. Studies in primary macrophages and liver strongly suggest an additional layer of tissueand context-specific regulation $[11,12]$. How the same receptor can simultaneously both activate and repress target genes remains an open and equally puzzling question. Tight control over which genes are activated and which are suppressed ensures proper execution of two of GR's signature functions: induction of glucose synthesis and suppression of inflammation.

This review discusses the recent insights into gene regulation by the GR in liver and macrophages and focuses on the genomic and epigenetic mechanisms that determine the cell type- and locus-specificity of GRmediated gene regulation.

\section{Who's talking? GR's neighborhood in hepatocytes and macrophages}

\subsection{GR acts as a master regulator of glucose metabolism in liver}

As a master player in maximizing circulating glucose output to maintain proper body function in times of fasting and stress, GR activates processes across different tissues which all serve the one purpose of providing substrates to the liver to fuel de novo gluconeogenesis [13]. In the liver, GR controls expression of phosphoenolpyruvate carboxykinase 1 (Pck1) and glucose-6-phosphatase (G6pc): two rate-limiting enzymes in hepatic gluconeogenesis. Functional GREs in promoters of both genes have been identified and characterized over the past 20 years and serve as the paradigm of transcriptional activation under GR control [14, 15]. By up-regulating Pck1 and G6pc expression, GR also indirectly drives hepatic fatty acid synthesis through the induction of hyperglycemia and hyperinsulinemia, two processes implicated in de novo lipogenesis [16]

Another GR target gene involved in hepatic lipid metabolism is the hairy and enhancer of split-1 (Hes1) gene. Lemke et al. demonstrated that a liverspecific knockout of GR leads to the induction of Hes1 gene expression and ameliorates steatotis in a fatty liver mouse model [17]. GR negatively interferes with Hes1 promoter activity through the recruitment of histone deacetylases. Apparently Hes1 needs to be silenced in order for GR to execute its regulatory function on genomic liver target genes [17]. This 
negative feedback by GR was further shown in mice with a conditional knockout of Hes1 [18]. Here, loss of hepatic Hes1 led to abnormal GRmediated target gene regulation and a metabolic phenotype; once again demonstrating that silencing of Hes 1 by GR is necessary for proper GC action [18].

Other GR-regulated target genes in liver include the circadian gene period circadian clock 1 (Per1) and the tyrosine aminotransferase (TAT) gene, which is exclusively expressed in hepatocytes. A glucocorticoid-responsive element in the Per1 promoter was shown to be indispensable for mRNA induction both in vitro and in vivo, thus tying GR to the maintenance of circadian rhythms in liver [19]. The TAT gene is another example of a metabolic enzyme under GR control [20]. In the liver, it catalyzes the rate-limiting conversion of tyrosine to 4-hydroxyphenylpyruvate, an important step in amino acid metabolism [21].

With the wealth of whole-genome Chromatin-Immunoprecipitation (ChIPSeq) data now available, it is becoming increasingly clear that GR's action in the liver goes way beyond glucose and lipid metabolism. Pathway and biological process analyses of GR-bound sequences (GBSs) in mouse liver [11] illustrate the myriad of direct GR binding events (Figure 1). In addition to gluconeogenesis and lipid metabolism, pathways regulated by GR are insulin signaling, PPAR signaling, bile acid metabolism and growth hormone receptor signaling, to name a few.

\subsubsection{Endowing competence for hepatocyte cell fate: Neighboring motifs in liver}

By motif enrichment analysis of ChIP-Seq data sets for GR in mouse liver, transcription factor footprints for CCAAT/enhancer binding protein ( $c / E B P)$, hepatic nuclear factor 4 (Hnf4), Onecut-1 (Oc1) and the Forkhead factor family (Fox) have been identified as significantly enriched together with GREs (Figure 2) [7, 11, 22]. c/EBP was previously shown to be indispensable for GR access and binding of the DNA in mouse livers [11]. Indeed, disruption of $c / E B P$ binding to the DNA itself led to loss of chromatin accessibility and interfered with GR recruitment and DNA binding [11]. Interestingly, Hnf4, Oc1 
as well as members of the Forkhead factor family all have crucial roles in liver development, thus supporting their role as lineage-determining factors. Whereas Hnf4 is essential for mammalian hepatocyte differentiation [23] and maintenance of hepatic gene expression [24], Oc1 (or Hnf6) controls early hepatoblast migration [25] and coordinates time-specific gene expression during liver development [26]. Intriguingly, FoxA2 (also called Hnf3) is suggested to be involved in the establishment of competence by opening up closed chromatin in liver-specific genes. Gualdi et al. demonstrated that hepatic differentiation from the gut endoderm is characterized by HNF3 occupancy at the albumin enhancer, which keeps the enhancer transcriptionally silent but open for additional factor binding and gene activation [27]. Moreover, Foxa1 and Foxa2 knockout mice fail to develop a liver bud, further corroborating their crucial role for the onset of hepatogenesis [28]. While the ChIP-Seq approach can determine the presence of these factors, it is lacking the resolution required for the accurate mapping of binding sites. ChIP-exo (ChIP-Seq coupled to exonuclease digestion) is a new technique that overcomes this limitation and allows for more precise determination of binding sites at almost single base pair resolution. Starick et al. made use of ChIP-exo to identify $O c 1$ and FoxA as the closest TF footprints to GREs in different cell lines [8]. Moreover, Lim et al. identified the same two motifs by ChIP-exo in mouse liver, supporting their association with GREs in the hepatic cistrome [7].

Besides their requirement in liver development, c/EBP [29], Hnf4 [30], FoxA2 [31] and FoxO1 [32] all enable recruitment of GR to liver-specific promoters and enhancers, such as the Pck1 locus [33].

\subsection{GR signaling in macrophage inflammation}

For over 60 years, patients have greatly benefited from the potent antiinflammatory effects of GCs, making them the most widely prescribed drugs in anti-inflammatory therapy. GCs bind GR in a variety of immune cells and stimulate anti-inflammatory actions that differ depending on the stimulus and disease model. In this context, macrophages are at the front line of the innate immune response and the major targets in the resolution of inflammation in 
sepsis and contact allergies [34, 35]. Importantly, cytokine and chemokine production is efficiently suppressed by GR in a transcription-dependent manner (Figure 1) [36].

In macrophages, GR activates expression of both anti-inflammatory genes such as glucocorticoid-induced leucine zipper (Tsc22d3/Gilz), dual specificity phosphatase/MAP kinase phosphatase 1 (Dusp1) or krueppel-like factors 2 and 9 (Klf2, Klf9), and circadian genes such as period circadian clock 1 (Per1). However, the crucial anti-inflammatory function of ligand-bound GR in macrophages is the repression of inflammatory genes. This latter class of GR targets encodes classic inflammatory cytokines such as interleukin 6 (II6), interleukin 1 alpha/beta (I/1a/b), chemokines such as chemokine (C-C motif) ligand 2 or $5(\mathrm{Ccl} 2, \mathrm{Ccl} 5)$ or chemokine (C-X-C motif) ligand 10 ( $\mathrm{Cxc} / 10)$ and other inflammatory mediators such as inducible nitric oxide synthase 2 (Nos2), the matrix metalloproteases 12 and 13 (Mmp12/13) and tumor necrosis factor (Tnf) [12, 34].

\subsubsection{Towards a myeloid lineage: Role of priming factors in macrophages}

Genome-wide analyses of GR binding events by ChIP-Seq confirmed GR recruitment to enhancers regulating inflammatory genes, but did not yield the anticipated insight into defined regulatory sequences discriminating between activating and repressing regulatory elements [12]. A closer look at cooccurring transcription factor binding sites identified Spleen focus forming virus (SFFV) Proviral Integration oncogene (Spi1 also known as Pu.1), c/EBP, jun proto-oncogene (cJun) and v-rel ReticuloEndotheLiosis viral oncogene homolog A (RelA also known as p65) as possible binding partners at enhancers of both activated and repressed genes in macrophages (Figure 2) [7, 12]. Interestingly, Pu.1 and c/EBP are essential for development of the myeloid lineage [37, 38]. Loss of Pu. 1 in hematopoietic stem cells completely blocks their self-renewing capabilities. Furthermore, Pu.1 remains expressed after lineage commitment of hematopoietic stem cells towards lymphoid and myeloid lineages and is required for myeloid maturation, but not for proliferation or survival [38]. Conversely, loss of $c / E B P \alpha$ increases the 
proliferation and self-renewing capabilities of hematopoietic stem cells, and blocks the M-CSF-dependent differentiation towards the myeloid lineage [39, 40]. Thus, both Pu.1 and c/EBP are required to balance hematopoietic stem cell proliferation versus differentiation, and have combinatorial functions in "priming" the myeloid lineage and establishing the monocyte-specific enhancer landscape [37, 41]. Additionally, other c/EBP isoforms, e.g. c/EBP $\beta$ and $\mathrm{c} / \mathrm{EBP \varepsilon}$ are required for myeloid differentiation [42] and inflammatory responses in macrophages [37]. In addition to their function in setting the myeloid enhancer landscape, a direct interaction of GR with c/EBPa in human primary cells was shown [43].

Furthermore, direct protein-protein interactions of GR with the NF-kB subunit p65 in Hela cells under overexpression conditions [44] or in vitro [45] and the AP-1 subunit c-Jun in vitro [46] was demonstrated. Additionally, in 2011 Biddie and colleagues showed that AP-1 (c-Jun/c-Fos heterodimer) is necessary for GR recruitment to a subset of AP-1/GR composite elements in 3134 cells [47]. Similarly, upon TNFalpha stimulation, Hela cells gain a subset of GREs associated with pro-inflammatory genes dependent on the presence of NF-kB /p65 by co-occupying those regulatory elements [48]. This strengthens the argument that GR-mediated gene regulation depends on the simultaneous action of AP-1/c-Jun and NF-kB/p65. Interestingly, a closer look at the repressed GR cistrome revealed the Interferon Regulatory Factor 3 (IRF3) as an additional binding partner that might be involved in GR repression in macrophages [12]. Interestingly, in 2005 Ogawa and colleagues proposed a mechanism by which GR interferes with the lipopolysaccharide (LPS)-induced p65/IRF3 interaction at a subset of shared enhancers [45].

Clearly, although a deeper insight into GR-mediated pro- and antiinflammatory gene regulation has been gained in recent years, much remains to be learned regarding the mechanisms underlying selective gene repression and activation by GC-bound GR in macrophages.

\section{GR and its coregulators}

Coregulators typically act via three distinct mechanisms to ultimately link upstream cellular signaling events from the environment into functional 
genomic responses: by remodeling chromatin in an ATP-dependent manner, by covalently modifying histones or other components of transcription complexes (or recruiting secondary cofactors with such activities), or by directly facilitating the assembly of basic transcriptional machinery. Interestingly, growing evidence suggests that coregulators function in a target gene-specific manner. Thus, although the exact mechanisms that dictate specificity of cofactor utilization by GR remain poorly defined, these proteins potentially represent attractive candidates that can be manipulated in order to bias GR towards specific regulatory programs. In the following section, we discuss recent studies pointing to less typical and perhaps unexpected coregulator activities in regards to GR-driven gene expression.

\subsection{Nuclear Receptor Coactivators (NCOAs) of the p160 family}

The most well characterized nuclear receptor coregulators are adaptor proteins of the p160 family, NCOA1/SRC1, NCOA2/TIF2/GRIP1/SRC2, and NCOA3/pCIP/ACTR/AIB1/SRC3. The importance for these proteins in diverse biological processes including metabolism, circadian rhythm, immunity and reproduction as revealed by gene ablation studies in mice has been extensively reviewed elsewhere [49-51].

All p160s are recruited by the AF2 regions of agonist-activated NRs via one of three LXXLL motifs (NR boxes; GR interacts preferentially with NR box-3) in their NR interaction domain (NID) [52, 53]. Acting as binding platforms for numerous secondary chromatin modifiers such as CBP1/p300, PRMT1, CARM1, the Baf57 subunit of the SWI/SNF complex and other cofactors, p160 family members ultimately potentiate the activation of associated target genes. Because much of the molecular characterization of the p160 coactivator functions as they relate specifically to GR has been performed using in vitro and overexpression systems, the involvement of individual p160s in driving transcription of GC-responsive genes and, hence, their contribution to specific GR-driven biological pathways in vivo, remains relatively unclear. Future genome-wide analyses of GR transcription programs using different cell types lacking p160s in question will be instrumental in 
linking a wealth of structural and biochemical data on p160 function to GCregulated physiological processes.

Notably, with respect to GR, one p160 family member - NCOA2 or GRinteracting protein 1 (GRIP1) - stands out. In addition to its coactivator properties, it is the only p160 family member to have emerged as a key GR corepressor at GR, AP-1 and NF-KB co-occupied sites [54, 55]. GRIP1 was found to be recruited to such sites in conjunction with liganded GR and potentiate repression of associated genes, e.g., Tnf, Ccl2, Cc/3, Cc/4, I/1a, $1 / 1 \mathrm{~b}$ and many others [12]. In fact, at the transcriptomic level, almost half of genes induced by inflammatory triggers such as LPS and repressed by liganded GR in murine bone marrow-derived macrophages (BMMФ) lost their GC sensitivity in GRIP1-deficient BMMФ [56].

The mechanistic basis of GR:GRIP1-mediated repression is not fully understood, however this activity appears to require a unique domain of GRIP1 not conserved across the p160 family [54]. Moreover, many genes susceptible to repression by GR:GRIP1 were found to be targeted at the postinitiation step. Historically, signal-dependent transcription factors and, their coregulators were thought to affect chromatin and components of basal transcriptional machinery ultimately facilitating or precluding RNA Polymerase II recruitment and ultimately transcription initiation [57]. Indeed, genes such as II1b and II1a are repressed by GR:GRIP1 via inhibition of Pol II recruitment and transcription initiation (Figure $3 A$, left) [58]. In recent years, however, an alternative picture has emerged whereby early transcription elongation represents a critical rate-limiting step for up to $40 \%$ of regulated genes [59, 60]. At such genes, Pol II initiates transcription prior to stimulation but pauses 50 nucleotides from the TSS bound by the Negative Elongation Factor (NELF) [59-62]. Upon exposure to relevant stimulus, positive transcription elongation factor P-TEFb, composed of cyclin T1 and CDK9, phosphorylates Pol II as well as NELF triggering its dissociation and the release of Pol II into productive elongation (Figure $3 A$, right). This level of regulation is very common among genes encoding inflammatory cytokines such as TNF, CCL3 and CCL2 [63], all of which are also targets for GR:GRIP1-mediated repression. Furthermore, it was shown in primary macrophages that at those genes, it was signal-dependent pause release that was attenuated by 
GR:GRIP1. Specifically, GR:GRIP1 complex inhibited P-TEFb recruitment and triggered NELF accumulation at the TSS (Figure $3 A$, right) [58, 63]. Consistent with a critical role of NELF in GR-mediated repression specifically of such 'elongation-controlled' genes, their response to Dex was blunted in NELF-deficient macrophages [58]. Importantly, GR-imposed control of pause release required GRIP1 as the attenuation of P-TEFb recruitment and the retention of NELF were both abrogated by GRIP1 deletion, thus rendering these pro-inflammatory genes GC-resistant [58]. This unexpected finding points to previously unappreciated function that GRIP1 and perhaps other coregulators may play in controlling gene expression at post-initiation steps.

\subsection{TRIP6}

TRIP6 is a member of the zyxin family with roles in focal adhesion, which contains LIM domains involved in protein:protein interactions. The nuclear isoform of TRIP6, nTRIP6, is thought to serve as an adaptor between GR and Fos-containing AP-1 dimers, facilitating hormone-dependent recruitment of GR to AP1 complexes in the context of "tethering" sites [64-66]. In the absence of hormone, nTRIP6 acted as an AP-1 coactivator by recruiting THRAP3 and MED1 subunits of the mediator complex (Figure 3B). Indeed, introduction of peptides that disrupt nTRIP6 dimerization and interaction with the mediator resulted in blunted recruitment of THRAP3 and MED1 to an AP1-driven Mmp13 promoter in response to phorbol ester (e.g., TPA) and transcriptional activation [65]. In the presence of GC, however, GR bound nTRIP6 thereby precluding the nTRIP6:THRAP3 and nTRIP6:MED1 interactions and attenuating gene activation in response to TPA (Figure 3B). The repressive effect of liganded GR was abrogated when nTRIP6 was depleted, although the extent to which gene repression by GR vs. gene activation by AP-1 was affected by TRIP6 knockdown is difficult to determine [67]. In addition, given that much of this work has been performed in cell lines with overexpression constructs, it will be essential to extend these studies to gene silencing with endogenous systems in inflammatory cell types to gauge their physiological relevance. 


\subsection{Hydrogen Peroxide-Inducible Clone 5 (HIC5)}

HIC5/TGFB1I1 is, like TRIP6 or Paxillin, a LIM domain protein involved in focal adhesion, that also serves as a coregulator for multiple NRs. HIC5 binds the $\square 2$ hinge domain of human GR [68] and can function to either potentiate or attenuate GR actions. The unique feature of HIC5, however, was revealed through genome-wide studies following HIC5 siRNA-mediated depletion in human osteosarcoma cells overexpressing GR, U2OS-GR [69]. In this study, about $40 \%$ of Dex-regulated genes were affected by HIC5 depletion, with the vast majority of those becoming less Dex-responsive (i.e., genes activated by Dex were less activated, and genes repressed by Dex were less repressed) in HIC5-deficient cells relative to wild-type. This is in agreement with conventional actions of HIC5 as a GR coregulator as ChIP-Seq revealed GBS near many of these candidate Dex-activated genes and ChIP-qPCR analysis showed that HIC5 depletion resulted in decreased recruitment of MED1 and Pol II to their TSS. The unexpected finding, however, was that a significant number of genes acquired Dex responsiveness only following HIC5 knockdown. Indeed, those so-called "blocked" genes displayed sites of Dexinduced GR occupancy as well as p300/CBP, MED1 and Pol II recruitment to the TSS that gained positive Dex regulation upon depletion of HIC5, which also correlated with greatly increased chromatin opening as assessed by FAIRE-qPCR (Figure 3C). These results illustrate a non-traditional role for HIC5 as a cofactor in that by selectively promoting chromatin remodeling, it dictates binding site selection by GR. The described scenario challenges an established model, according to which cofactors are passively recruited to chromatin by DNA-bound regulators. However, its physiological role is so far unknown and presents a fascinating topic for future studies.

HIC5 interacts with multiple steroid receptors; interestingly, however, despite binding GRa (the major GR isoform), GRy and ERa similarly, its depletion affected the greatest number of GRa-regulated genes [70]. Still, "blocked" genes, whose increased chromatin accessibility and hormonal regulation was unveiled through HIC5 knockdown, were found for all steroid receptors analyzed. These studies therefore point to a previously unappreciated active function of coregulators in dictating the specificity of 
transcription factor actions. Indeed, for some factors for which genome-wide data are available, there appear to be vastly more potential binding sites present than is actually being utilized (e.g., AP-1) [71], and what determines tissue-specific binding remains poorly understood. A role for coregulators in limiting chromatin accessibility to GR or other factors represents a compelling mechanism that would enable binding site selection to operate in a cellspecific manner and, thus, an exciting new avenue for future studies.

\section{Shaping cell type specification}

\subsection{Role of the epigenetic landscape in GR gene regulation}

The epigenetic landscape of a cell is a major contributor to cell typespecific responses upon stimulation. GCs have long been known to elicit tissue-specific effects. Whereas the physiological function of GCs in providing glucose for energy production is mainly mediated by hepatocytes, their most relevant function in clinical practice lies in the anti-inflammatory actions in immune cells.

Target genes of the ligand-bound GR form two groups according to their cell type-specific behavior. The first group is composed of genes, which are regulated by GR independently of cellular context like the maintenance of basal cellular processes or circadian effects, e.g. Per1 or Klf9 [11, 12, 72]. The second group includes genes with their expression changing in some but not in other tissues upon GC treatment, e.g. the metabolic tissue-specific genes Pck1 [73] and G6pc [74, 75] or the inflammatory cytokines $I / 6$ and I/1a, which are repressed by GR in macrophages [12], but not in hepatocytes.

ChIP-Seq experiments on GR have revealed that GR binds different enhancers depending on the cell type. It was for example shown that GR binds the enhancer and promoter regions of Pck1 and G6pc in liver [11], but not in macrophages treated with LPS. On the other hand, GR binds the enhancers of $1 / 6$ and I/1a in macrophages after LPS treatment [12] but not in hepatocytes (Figure 4). A closer look at the epigenetic signature revealed that both the Pck1 and G6pc enhancer are acetylated at H3K27 and monomethylated at H3K4 in hepatocytes [76] but not in activated macrophages [57]. The same applies to the enhancers of $1 / 6$ and $/ 11$ a. Indeed, 
up to a third of GR-bound sites in liver and macrophages overlap with H3K4me1 and H3K27Ac chromatin that is modified in the absence of GR and its ligand. (Figure 4) [11, 12, 57, 76]. This indicates the requirement of active enhancers [77] and open chromatin for GR binding in both liver [11] and macrophages. Similarly, results from John and colleagues demonstrated that GR requires "open" chromatin for binding in 3134 and AtT-20 cells [78]. Therefore, cell type-specific actions of GR appear to depend on the epigenetic landscape and DNA accessibility rather than on chromatin remodeling and enhancer priming by GR itself.

Motif prediction analysis in GR ChIP-Seq experiments revealed that Pu.1 and c/EBP are among the most abundant motifs co-localizing with GREs in macrophages (Figure 2) [12] whereas in liver, GREs co-occur with c/EBP, Hnf4, Oc1 and Forkhead motifs (Figure 2) [11]. Pu.1 and c/EBP in macrophages as well as Hnf4, Oc1, c/EBP and Forkhead proteins in hepatocytes, respectively, are required for the development of those lineages, thus defining them as lineage-determining factors [23-28, 37-41]. Closer analysis of the combinatorial requirements of transcription factors for lineage specification and integration of cell type-specific signal responses in macrophages was consistent with a hierarchical mechanism underlying transcription factor networks [79, 80]. First, lineage-determining factors establish the basic cell type-dependent accessibility of the DNA, followed by integration of environmental and, lastly, temporal stimuli (Figure 2) [81, 82].

In conclusion, the cell type-specificity of the GR transcriptome is a consequence of lineage-specific enhancer accessibility and activity. The diversity in GR responses is determined by the cooperativity of cell type-, environment- or signal-dependent transcription factors. Studying how the temporally constrained or continuous actions of these factors influence enhancer remodeling, GR occupancy and the recruitment of coregulators will yield important insights in years to come.

\subsection{Coregulator specificity towards certain physiological pathways}

Gene regulation by GCs is pleiotropic and affects diverse physiological pathways. Coregulators have long been thought to impart a level of specificity 
to GR transcriptional regulation, albeit it was attributed primarily to tissuespecific coregulator expression. More recently, a combination of genome-wide transcriptomics combined with systematic depletion of individual coregulators suggested that distinct GC-driven physiological pathways within the same cell type display differential cofactor requirements. Indeed, siRNA-mediated depletion of CCAR1, and not other 9 coregulators analyzed in 3T3-L1 murine pre-adipocytes, greatly attenuated Dex induction of adipogenic genes but not of select anti-inflammatory genes [83]. Consistently, knockdown of CCAR1 by shRNA in mouse mesenchymal stem cells (MSCs) blunted adipocyte differentiation in response to the adipogenic cocktail Dex/insulin/ibmx/rosiglitazone. To tease out the effects of CCAR1 resulting specifically from its cooperativity with GR, Dex only treatment was used to identify major regulatory GBS in the PPARY gene, a master regulator of adipocyte differentiation. ChIP- and FAIRE-qPCR analysis in MSCs revealed that GR and CCAR1 were recruited to these GBS in conjunction with chromatin opening at the PPARy promoter - all of which was abrogated by CCAR1 depletion. In this regard, it would be informative to evaluate the phenotype of adipocyte-specific CCAR1 knockout mice in vivo.

A similar genome-wide analysis of the Dex-responsive transcriptome was performed in A549 cells following siRNA-mediated depletion of either CCAR1, CCAR2, CALCOCO1 or ZNF282 [84]. Not surprisingly, the vast majority of genes affected by coregulator depletion displayed decreased induction by Dex, with ZNF282 knockdown affecting the greatest number of genes ( 3000, $3 x$ more than the others). Of these genes, 1/3 were factor-specific with distinct polarity of regulation: CCAR1- and 2-unique genes induced by Dex were attenuated by their depletion - supporting the role of CCAR1 and 2 as GR coactivators; conversely, CoCoA- and ZNF282-unique genes induced by Dex were superactivated by their knockdown suggesting that CoCoA- and ZNF282 inhibit GR activity. Similar to the HIC5 studies, this experiment revealed "blocked" genes that became Dex-regulated only after cofactor depletion concomitantly with the loss of the repressive histone mark H3K27me3, suggesting a role for these coregulators in limiting chromatin accessibility to GR (Figure 3C). Interestingly, "blocked" genes were virtually all coregulatorspecific and the majority (50-75\%) gained positive regulation by Dex. Given 
the therapeutic utility of GCs, Ingenuity Pathway Analysis (IPA) was used to assess the impact of different cofactors on pathways that contribute to the anti-inflammatory effects of Dex. IPA of the acute phase response supported a significant role of ZNF282 and CoCoA in GR-mediated repression of the NF-KB pathway as well as in limiting glucocorticoid induction of STAT3, and of ZNF282 in glucocorticoid repression of c-Jun and the JNK pathway. In contrast, all coregulators but CCAR2 appeared to have similar effects on interferon signaling by limiting the Dex-induced expression of JAK1 and STAT1. CCAR2 was the exception, as IPA suggested that it potentiated Dexmediated inhibition of interferon receptor expression. This limited analysis already reveals the ability of GR coregulators to preferentially participate in specific physiological pathways. It will be interesting to see this type of characterization extended to other cell types and tissues, such as those with key role in inflammation and metabolism given the dire need for more selective GC-like molecules in clinical practice.

\section{Future directions}

Recent genome-wide studies have provided unprecedented insights into the molecular and genomic mechanisms of GR action. Despite the increasing identification of binding sites, target genes and coregulators, however, we are only beginning to decipher GR's distinctive actions across the different cell types.

High-resolution mapping of GBS with new next generation sequencing techniques such as ChIP-exo as well as advanced bioinformatic analyses will yield additional clues on the genomic information that specifies enhancers. Furthermore, the decoding of locus-specific GR/cofactor complexes by highresolution mass spectrometry will deepen our understanding of the combinatorial complexity at GR-regulated enhancers and the role of signal integration via post-translational modifications. An emerging role of long noncoding RNAs in gene regulation provides an additional layer of complexity as does the 3-dimensional architecture of the nucleus; two aspects which so far have been insufficiently studied in the context of GR action. Furthermore, studies in large non-synchronized cell populations and heterogeneous tissues 
merely provide a cumulative snapshot of the underlying molecular events. Here, differences between individual cells based on cell cycle, environmental signals and positional information provided by signaling gradients or tissue architecture are neglected. With the continuous advancement of both next generation sequencing and high resolution imaging techniques towards higher sensitivity and smaller scales, we can anticipate that single cell analyses, in particular in-situ approaches, will further deepen our understanding of GC signaling in the near future.

\section{Acknowledgments}

We apologize to all authors whose work could not be cited due to the limited scope of this review. We thank Dr. Y. Chinenov for preparation of the figures. This work was supported by grants UH 275/1-1 (DFG) to FG, MCH and NHU; 638573 SILENCE (ERC-2014-StG) to NHU; a Daimler Benz Scholarship to FG; the NIH/NIDDK (R01 DK099087), The Department of Defense (PR130049) and The Rheumatology Research Foundation to IR and the NIH/NIAMS T32 AR007281 to DAR. 


\section{Figure legends}

Figure 1: Cell type-specific effects of GCs in liver and macrophages. The GR stimulates gluconeogenesis, fatty acid (FA) synthesis and cholesterol transport in liver, whereas it represses lipid storage, B-oxidation and bile acid synthesis favoring the release of glucose for a fast energy provision. Signaling pathways affected by GR are Peroxisome Proliferator Activated Receptor (PPAR) and growth hormone receptor (GhR) signaling. The main function of GR in macrophages is its repressive actions on inflammation via inhibition of cytokine production, suppression of interleukin 6 (II6) and lipopolysaccharide (LPS) signaling. Circadian rhythm is influenced by glucocorticoids in both tissues. $\rightarrow$ activation; -| repression

Fiqure 2: Mechanism of cell type-specific gene regulation by GR. (A) Lineage-determining factors (LDFs) are transcription factors that recognize their DNA binding motif (LRE) within compacted chromatin and recruit chromatin remodeling machinery to "open" it and to reposition nucleosomes. Subsequent recruitment of histone demethylases/methylases and histone acetyltransferases establishes a cell type-specific enhancer landscape. "Open" chromatin is marked by increased DNA accessibility and specific histone marks, one being acetylation at H3K27, replacing a methylation mark at this residue (H3K27me3) associated with repressed chromatin. Enhancers are marked by a monomethylation mark at $\mathrm{H} 3 \mathrm{~K} 4$, and active enhancers harbor the double label H3K4me1/H3K27ac. Once the enhancer landscape of a cell is specified, signal-dependent transcription factors such as GR are able to access their response elements (GREs). Together with co-occuring transcription factors (coTF), they regulate target genes in a locus-specific manner. The combinatorial diversity of GREs and TFREs (response elements of co-occuring transcription factors) is the hallmark of gene-specific outcomes of GR signaling. (B) Transcription factor binding motifs identified as cooccuring with GREs in ChIP-Seq experiments in liver and macrophages can be classified as LREs or TFREs. 
Figure 3: Mechanisms of GR coregulator function A. GR:GRIP1 repress initiation- and elongation-controlled genes by distinct mechanisms. Initiationcontrolled genes: GR:GRIP1 complex blocks the recruitment of Pol II to the TSS, the rate-limiting step for gene activation. Elongation-controlled genes: GR:GRIP1 complex blunts CDK9 recruitment, and promotes accumulation of NELF at the TSS thus blocking phosphorylation of Serine 2 of the Pol II Cterminal domain and entry into productive elongation. B. nTRIP6-facilitated repression by GR. In the presence of activating signals such as TPA, nTRIP6 acts as a scaffold for the recruitment of THRAP3 and the Mediator complex to Fos-containing AP-1 dimers thus potentiating activation of associated target genes. Upon GC exposure, GR binds to nTRIP6 and precludes its interaction with THRAP3 and Mediator, thereby attenuating target gene transcription. C. Coregulators dictate $\mathrm{GR}$ binding site selection. In the presence of the coregulators HIC5, ZNF282, CCAR1/2, or CALCOA1, a subset of potential GREs are associated with inaccessible chromatin and the repressive histone mark, H3K27me3. Upon coregulator depletion, chromatin accessibility increases and GR can bind the open binding sites and regulate transcription of associated genes.

Figure 4: Chromatin accessibility mediates cell type-specific actions of GR. ChIP-Seq tracks for GR, H3K4me3, H3K4me1 and H3K27ac in liver and inflammatory macrophages. (A) For Per1, activated by GR in both tissues, the GR peaks lie in close proximity to H3K4 monomethylated chromatin - a histone mark associated with enhancers. This enhancer is active in hepatocytes (overlapping H3K27ac) or poised in macrophages (missing H3K27ac). (B) In hepatocytes but not macrophages, GR binds close to the Pck1 promoter. The surrounding chromatin harbors the H3K4me1/ H3K27ac double mark for active enhancers as well as a H3K4 trimethyation mark, indicating the proximity to the Pck1 promoter. Macrophage chromatin at this locus contains neither enhancer/promoter-specific histone marks nor a GBS. (C) The 116 enhancer contains a GBS in macrophages, which overlaps with H3K4me1/H3K27ac chromatin, indicating an active enhancer. In hepatocytes this chromatin is not accessible for GR binding and harbors none of these marks. 


\section{References}

1. Evans, R.M., The steroid and thyroid hormone receptor superfamily. Science, 1988. 240(4854): p. 889-95.

2. Mangelsdorf, D.J., et al., The nuclear receptor superfamily: the second decade. Cell, 1995. 83(6): p. 835-9.

3. Vegiopoulos, A. and S. Herzig, Glucocorticoids, metabolism and metabolic diseases. Mol Cell Endocrinol, 2007. 275(1-2): p. 43-61.

4. Cole, T.J., et al., Targeted disruption of the glucocorticoid receptor gene blocks adrenergic chromaffin cell development and severely retards lung maturation. Genes Dev, 1995. 9(13): p. 1608-21.

5. Schacke, H., W.D. Docke, and K. Asadullah, Mechanisms involved in the side effects of glucocorticoids. Pharmacol Ther, 2002. 96(1): p. 2343.

6. Shibli-Rahhal, A., M. Van Beek, and J.A. Schlechte, Cushing's syndrome. Clin Dermatol, 2006. 24(4): p. 260-5.

7. Lim, H.W., et al., Genomic redistribution of GR monomers and dimers mediates transcriptional response to exogenous glucocorticoid in vivo. Genome Res, 2015. 25(6): p. 836-44.

8. $\quad$ Starick, S.R., et al., ChIP-exo signal associated with DNA-binding motifs provides insight into the genomic binding of the glucocorticoid receptor and cooperating transcription factors. Genome Res, 2015. 25(6): p. 825-35.

9. Glass, C.K. and K. Saijo, Nuclear receptor transrepression pathways that regulate inflammation in macrophages and T cells. Nat Rev Immunol, 2010. 10(5): p. 365-76.

10. Langlais, D., et al., The Stat3/GR interaction code: predictive value of direct/indirect DNA recruitment for transcription outcome. Mol Cell, 2012. 47(1): p. 38-49.

11. Grontved, L., et al., C/EBP maintains chromatin accessibility in liver and facilitates glucocorticoid receptor recruitment to steroid response elements. EMBO J, 2013. 32(11): p. 1568-83.

12. Uhlenhaut, N.H., et al., Insights into negative regulation by the glucocorticoid receptor from genome-wide profiling of inflammatory cistromes. Mol Cell, 2013. 49(1): p. 158-71.

13. Patel, R., J. Williams-Dautovich, and C.L. Cummins, Minireview: new molecular mediators of glucocorticoid receptor activity in metabolic tissues. Mol Endocrinol, 2014. 28(7): p. 999-1011.

14. Imai, E., et al., Characterization of a complex glucocorticoid response unit in the phosphoenolpyruvate carboxykinase gene. Mol Cell Biol, 1990. 10(9): p. 4712-9.

15. Vander Kooi, B.T., et al., The glucose-6-phosphatase catalytic subunit gene promoter contains both positive and negative glucocorticoid response elements. Mol Endocrinol, 2005. 19(12): p. 3001-22.

16. Sun, Z. and M.A. Lazar, Dissociating fatty liver and diabetes. Trends Endocrinol Metab, 2013. 24(1): p. 4-12.

17. Lemke, U., et al., The glucocorticoid receptor controls hepatic dyslipidemia through Hes1. Cell Metab, 2008. 8(3): p. 212-23. 
18. Revollo, J.R., et al., HES1 is a master regulator of glucocorticoid receptor-dependent gene expression. Sci Signal, 2013. 6(304): p. ra103.

19. Yamamoto, T., et al., Acute physical stress elevates mouse period1 mRNA expression in mouse peripheral tissues via a glucocorticoidresponsive element. J Biol Chem, 2005. 280(51): p. 42036-43.

20. Jantzen, H.M., et al., Cooperativity of glucocorticoid response elements located far upstream of the tyrosine aminotransferase gene. Cell, 1987. 49(1): p. 29-38.

21. Natt, E., et al., Point mutations in the tyrosine aminotransferase gene in tyrosinemia type II. (0027-8424 (Print)).

22. Phuc Le, P., et al., Glucocorticoid receptor-dependent gene regulatory networks. PLoS Genet, 2005. 1(2): p. e16.

23. Li, J., G. Ning, and S.A. Duncan, Mammalian hepatocyte differentiation requires the transcription factor HNF-4alpha. Genes Dev, 2000. 14(4): p. 464-74.

24. Hayhurst, G.P., et al., Hepatocyte nuclear factor 4alpha (nuclear receptor 2A1) is essential for maintenance of hepatic gene expression and lipid homeostasis. Mol Cell Biol, 2001. 21(4): p. 1393-403.

25. Margagliotti, S., et al., The Onecut transcription factors HNF-6/OC-1 and $\mathrm{OC}-2$ regulate early liver expansion by controlling hepatoblast migration. Dev Biol, 2007. 311(2): p. 579-89.

26. Beaudry, J.B., et al., Threshold levels of hepatocyte nuclear factor 6 (HNF-6) acting in synergy with HNF-4 and PGC-1alpha are required for time-specific gene expression during liver development. Mol Cell Biol, 2006. 26(16): p. 6037-46.

27. Gualdi, R., et al., Hepatic specification of the gut endoderm in vitro: cell signaling and transcriptional control. Genes Dev, 1996. 10(13): p. 1670-82.

28. Lee, C.S., et al., The initiation of liver development is dependent on Foxa transcription factors. Nature, 2005. 435(7044): p. 944-7.

29. Park, E.A., et al., Relative roles of CCAAT/enhancer-binding protein beta and $C A M P$ regulatory element-binding protein in controlling transcription of the gene for phosphoenolpyruvate carboxykinase (GTP). J Biol Chem, 1993. 268(1): p. 613-9.

30. Hall, R.K., F.M. Sladek, and D.K. Granner, The orphan receptors COUP-TF and HNF-4 serve as accessory factors required for induction of phosphoenolpyruvate carboxykinase gene transcription by glucocorticoids. Proc Natl Acad Sci U S A, 1995. 92(2): p. 412-6.

31. Wang, J.C., et al., Hepatic nuclear factor 3 is an accessory factor required for the stimulation of phosphoenolpyruvate carboxykinase gene transcription by glucocorticoids. Mol Endocrinol, 1996. 10(7): p. 794-800.

32. Hall, R.K., et al., Regulation of phosphoenolpyruvate carboxykinase and insulin-like growth factor-binding protein-1 gene expression by insulin. The role of winged helix/forkhead proteins. J Biol Chem, 2000. 275(39): p. 30169-75.

33. Chakravarty, K., et al., Factors that control the tissue-specific transcription of the gene for phosphoenolpyruvate carboxykinase-C. Crit Rev Biochem Mol Biol, 2005. 40(3): p. 129-54. 
34. Kleiman, A., et al., Glucocorticoid receptor dimerization is required for survival in septic shock via suppression of interleukin-1 in macrophages. FASEB J, 2012. 26(2): p. 722-9.

35. Tuckermann, J.P., et al., Macrophages and neutrophils are the targets for immune suppression by glucocorticoids in contact allergy. J Clin Invest, 2007. 117(5): p. 1381-90.

36. Tuckermann, J.P., et al., Molecular mechanisms of glucocorticoids in the control of inflammation and lymphocyte apoptosis. Crit Rev Clin Lab Sci, 2005. 42(1): p. 71-104.

37. Heinz, S., et al., Simple combinations of lineage-determining transcription factors prime cis-regulatory elements required for macrophage and B cell identities. Mol Cell, 2010. 38(4): p. 576-89.

38. Iwasaki, H., et al., Distinctive and indispensable roles of PU.1 in maintenance of hematopoietic stem cells and their differentiation. Blood, 2005. 106(5): p. 1590-600.

39. Heath, V., et al., C/EBPalpha deficiency results in hyperproliferation of hematopoietic progenitor cells and disrupts macrophage development in vitro and in vivo. Blood, 2004. 104(6): p. 1639-47.

40. Hohaus, S., et al., PU.1 (Spi-1) and C/EBP alpha regulate expression of the granulocyte-macrophage colony-stimulating factor receptor alpha gene. Mol Cell Biol, 1995. 15(10): p. 5830-45.

41. Jin, F., et al., PU.1 and C/EBP(alpha) synergistically program distinct response to NF-kappaB activation through establishing monocyte specific enhancers. Proc Natl Acad Sci U S A, 2011. 108(13): p. 52905.

42. Akagi, T., et al., In vivo deficiency of both C/EBPbeta and C/EBPepsilon results in highly defective myeloid differentiation and lack of cytokine response. PLoS One, 2010. 5(11): p. e15419.

43. Rudiger, J.J., et al., Interaction of C/EBPalpha and the glucocorticoid receptor in vivo and in nontransformed human cells. Faseb j, 2002. 16(2): p. 177-84.

44. Ray, A. and K.E. Prefontaine, Physical association and functional antagonism between the $p 65$ subunit of transcription factor NF-kappa B and the glucocorticoid receptor. Proc Natl Acad Sci U S A, 1994. 91(2): p. 752-6.

45. Ogawa, S., et al., Molecular determinants of crosstalk between nuclear receptors and toll-like receptors. Cell, 2005. 122(5): p. 707-21.

46. Diamond, M.I., et al., Transcription factor interactions: selectors of positive or negative regulation from a single DNA element. Science, 1990. 249(4974): p. 1266-72.

47. Biddie, S.C., et al., Transcription factor AP1 potentiates chromatin accessibility and glucocorticoid receptor binding. Mol Cell, 2011. 43(1): p. 145-55.

48. Rao, N.A., et al., Coactivation of GR and NFKB alters the repertoire of their binding sites and target genes. Genome Res, 2011. 21(9): p. 1404-16.

49. Dasgupta, S., D.M. Lonard, and B.W. O'Malley, Nuclear receptor coactivators: master regulators of human health and disease. Annu Rev Med, 2014. 65: p. 279-92. 
50. Dasgupta, S. and B.W. O'Malley, Transcriptional coregulators: emerging roles of SRC family of coactivators in disease pathology. $\mathrm{J}$ Mol Endocrinol, 2014. 53(2): p. R47-59.

51. Rollins, D.A., M. Coppo, and I. Rogatsky, Minireview: nuclear receptor coregulators of the 160 family: insights into inflammation and metabolism. Mol Endocrinol, 2015. 29(4): p. 502-17.

52. Darimont, B.D., et al., Structure and specificity of nuclear receptorcoactivator interactions. Genes Dev, 1998. 12(21): p. 3343-56.

53. Heery, D.M., et al., A signature motif in transcriptional co-activators mediates binding to nuclear receptors. Nature, 1997. 387(6634): p. 733-6.

54. Rogatsky, I., et al., Alternate surfaces of transcriptional coregulator GRIP1 function in different glucocorticoid receptor activation and repression contexts. Proc Natl Acad Sci U S A, 2002. 99(26): p. 167016.

55. Rogatsky, I., K.A. Zarember, and K.R. Yamamoto, Factor recruitment and TIF2/GRIP1 corepressor activity at a collagenase-3 response element that mediates regulation by phorbol esters and hormones. Embo j, 2001. 20(21): p. 6071-83.

56. Chinenov, Y., et al., Glucocorticoid receptor coordinates transcription factor-dominated regulatory network in macrophages. BMC Genomics, 2014. 15: p. 656.

57. Ostuni, R., et al., Latent enhancers activated by stimulation in differentiated cells. Cell, 2013. 152(1-2): p. 157-71.

58. Gupte, R., et al., Glucocorticoid receptor represses proinflammatory genes at distinct steps of the transcription cycle. Proc Natl Acad Sci U S A, 2013. 110(36): p. 14616-21.

59. Muse, G.W., et al., RNA polymerase is poised for activation across the genome. Nat Genet, 2007. 39(12): p. 1507-11.

60. Zeitlinger, J., et al., RNA polymerase stalling at developmental control genes in the Drosophila melanogaster embryo. Nat Genet, 2007. 39(12): p. 1512-6.

61. Jonkers, I. and J.T. Lis, Getting up to speed with transcription elongation by RNA polymerase II. Nat Rev Mol Cell Biol, 2015. 16(3): p. 167-77.

62. Peterlin, B.M. and D.H. Price, Controlling the elongation phase of transcription with P-TEFb. Mol Cell, 2006. 23(3): p. 297-305.

63. Adelman, K., et al., Immediate mediators of the inflammatory response are poised for gene activation through RNA polymerase II stalling. Proc Natl Acad Sci U S A, 2009. 106(43): p. 18207-12.

64. Diefenbacher, M., et al., Restriction to Fos family members of Trip6dependent coactivation and glucocorticoid receptor-dependent transrepression of activator protein-1. Mol Endocrinol, 2008. 22(8): p. 176780.

65. Diefenbacher, M.E., et al., The LIM domain protein nTRIP6 recruits the mediator complex to AP-1-regulated promoters. PLoS One, 2014. 9(5): p. e97549.

66. Kassel, O., et al., A nuclear isoform of the focal adhesion LIM-domain protein Trip6 integrates activating and repressing signals at AP-1- and NF-kappaB-regulated promoters. Genes Dev, 2004. 18(20): p. 2518-28. 
67. Diefenbacher, M.E., et al., The nuclear isoform of the LIM domain protein Trip6 integrates activating and repressing signals at the promoter-bound glucocorticoid receptor. Mol Cell Endocrinol, 2010. 320(1-2): p. 58-66.

68. Yang, L., et al., Interaction of the tau2 transcriptional activation domain of glucocorticoid receptor with a novel steroid receptor coactivator, Hic5, which localizes to both focal adhesions and the nuclear matrix. Mol Biol Cell, 2000. 11(6): p. 2007-18.

69. Chodankar, R., et al., Hic-5 is a transcription coregulator that acts before and/or after glucocorticoid receptor genome occupancy in a gene-selective manner. Proc Natl Acad Sci U S A, 2014. 111(11): p. 4007-12.

70. Chodankar, R., et al., Selective coregulator function and restriction of steroid receptor chromatin occupancy by Hic-5. Mol Endocrinol, 2015. 29(5): p. 716-29.

71. Zhou, H., et al., Frequency and distribution of AP-1 sites in the human genome. DNA Res, 2005. 12(2): p. 139-50.

72. Wiench, M., et al., DNA methylation status predicts cell type-specific enhancer activity. EMBO J, 2011. 30(15): p. 3028-39.

73. Hanson, R.W. and L. Reshef, Regulation of phosphoenolpyruvate carboxykinase (GTP) gene expression. Annu Rev Biochem, 1997. 66: p. 581-611.

74. Lin, B., D.W. Morris, and J.Y. Chou, Hepatocyte nuclear factor 1alpha is an accessory factor required for activation of glucose-6-phosphatase gene transcription by glucocorticoids. DNA Cell Biol, 1998. 17(11): p. 967-74.

75. Schmoll, D., B.B. Allan, and A. Burchell, Cloning and sequencing of the $5^{\prime}$ region of the human glucose-6-phosphatase gene: transcriptional regulation by cAMP, insulin and glucocorticoids in H4IIE hepatoma cells. FEBS Lett, 1996. 383(1-2): p. 63-6.

76. Yue, F., et al., A comparative encyclopedia of DNA elements in the mouse genome. Nature, 2014. 515(7527): p. 355-64.

77. Creyghton, M.P., et al., Histone H3K27ac separates active from poised enhancers and predicts developmental state. Proc Natl Acad Sci U S A, 2010. 107(50): p. 21931-6.

78. John, S., et al., Chromatin accessibility pre-determines glucocorticoid receptor binding patterns. Nat Genet, 2011. 43(3): p. 264-8.

79. Gosselin, D., et al., Environment drives selection and function of enhancers controlling tissue-specific macrophage identities. Cell, 2014. 159(6): p. 1327-40.

80. Lavin, Y., et al., Tissue-resident macrophage enhancer landscapes are shaped by the local microenvironment. Cell, 2014. 159(6): p. 1312-26.

81. Kaikkonen, M.U., et al., Remodeling of the enhancer landscape during macrophage activation is coupled to enhancer transcription. Mol Cell, 2013. 51(3): p. 310-25.

82. Zhang, D.X. and C.K. Glass, Towards an understanding of cell-specific functions of signal-dependent transcription factors. J Mol Endocrinol, 2013. 51(3): p. T37-50.

83. Ou, C.Y., et al., Coregulator cell cycle and apoptosis regulator 1 (CCAR1) positively regulates adipocyte differentiation through the 
glucocorticoid signaling pathway. J Biol Chem, 2014. 289(24): p. 17078-86.

84. Wu, D.Y., et al., Distinct, genome-wide, gene-specific selectivity patterns of four glucocorticoid receptor coregulators. Nucl Recept Signal, 2014. 12: p. e002. 


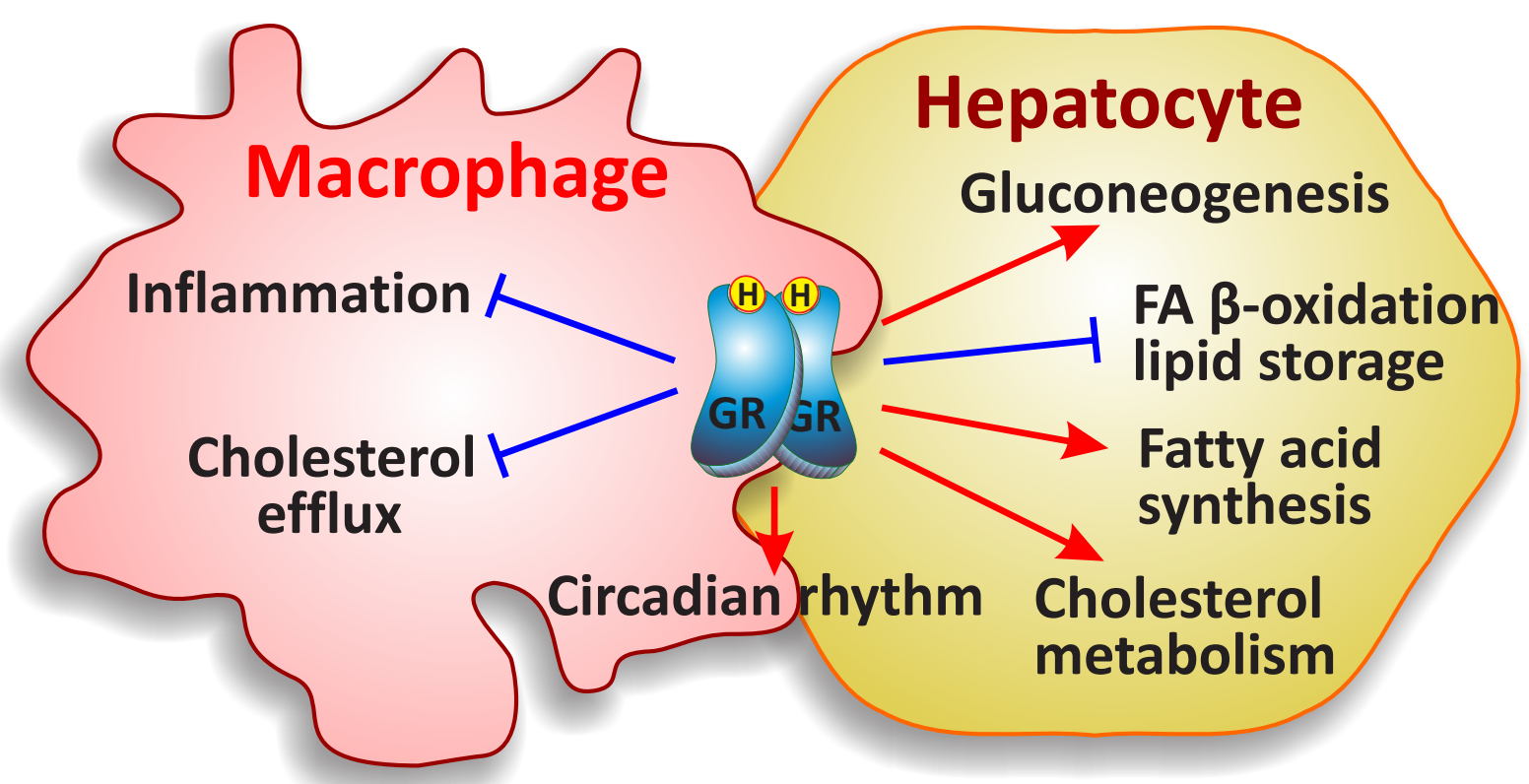


A

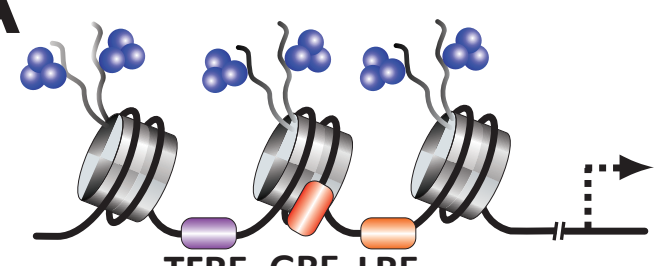

TFRE GRE LRE
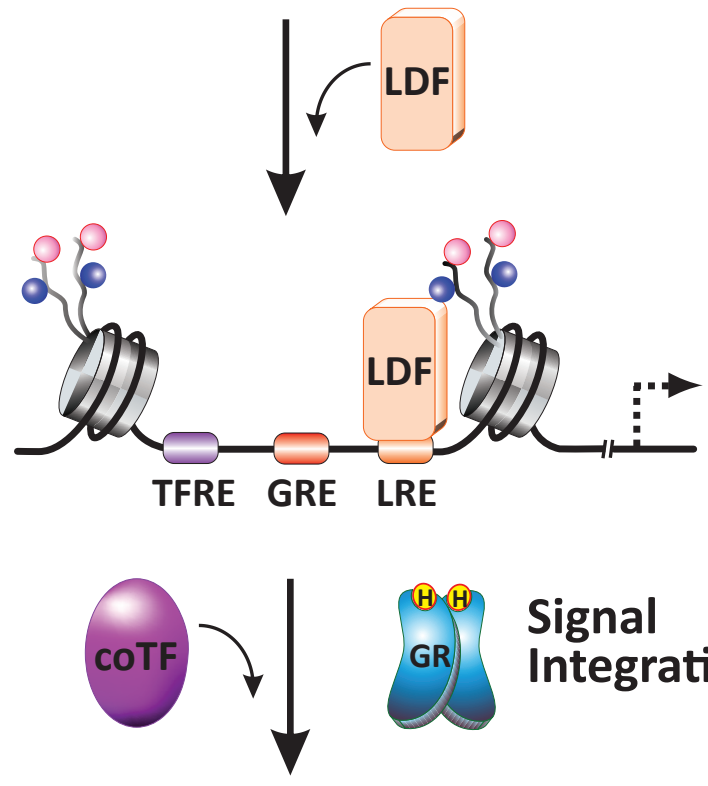

Signal Integration

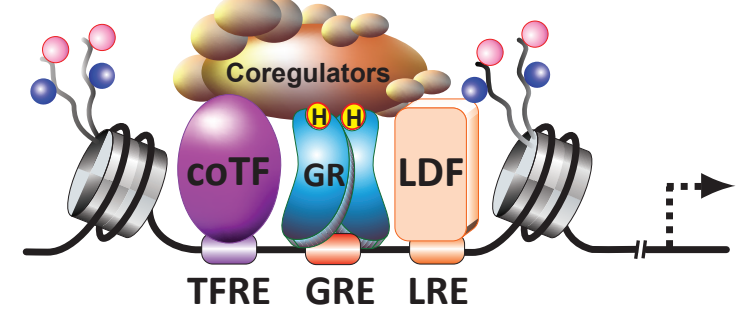

8. H3 K27Me3 Он3 К27Ac ОН3 K4Me1
B

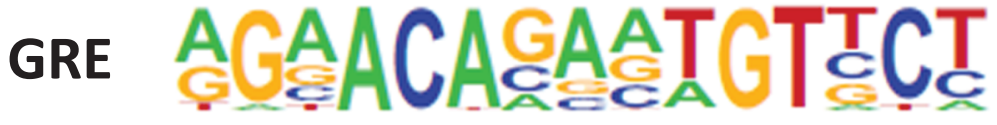

LDF - lineage determining factors Pu.1 AGAGGAAGT c/EBP ATTGCCAAC

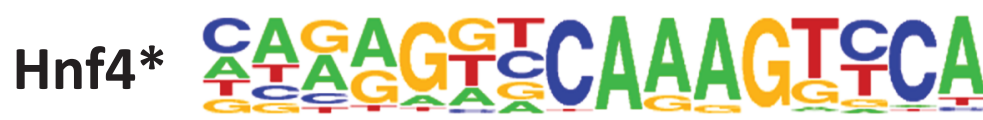
Oct1*

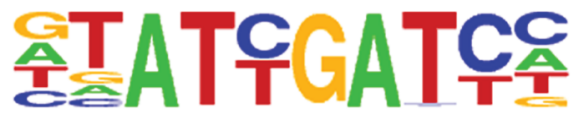
Forkhead* ¿TAAAcAA coTF - co-occuring transcr. factors AP-1/c-Jun ATGACTCATC NF-kB/p65 술GGATTTCCC 
A AP1/
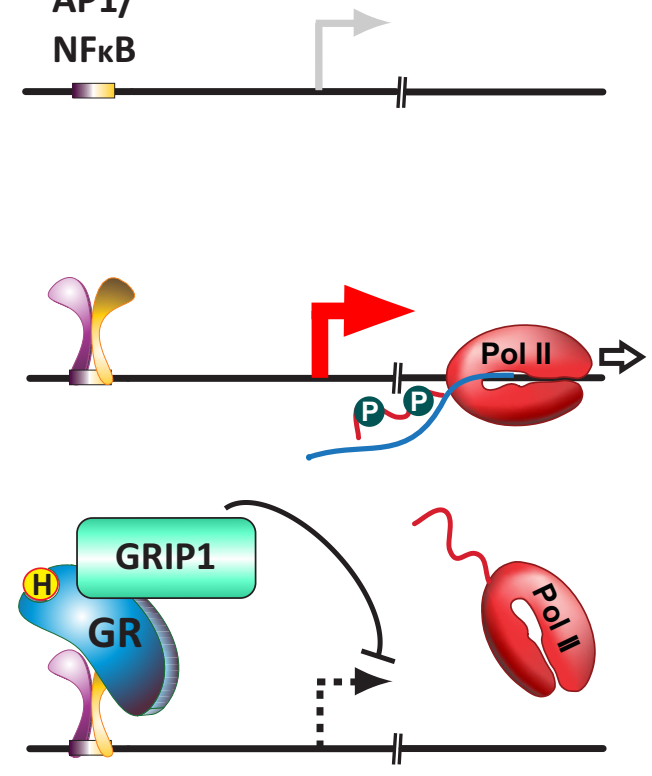

Initiation-controlled genes

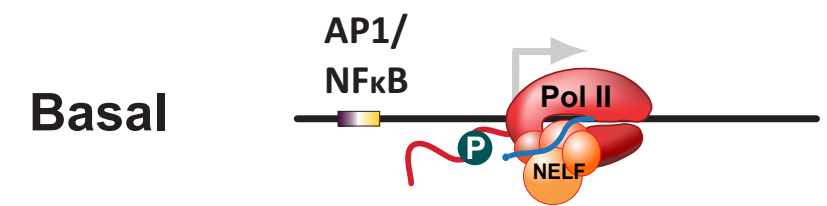

Induced

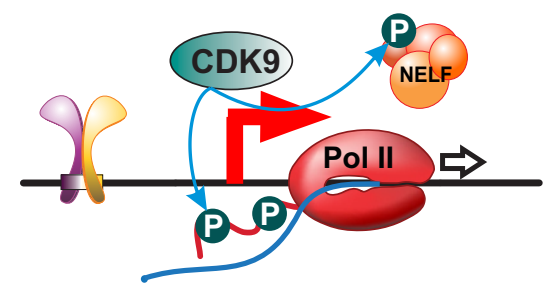

Repressed

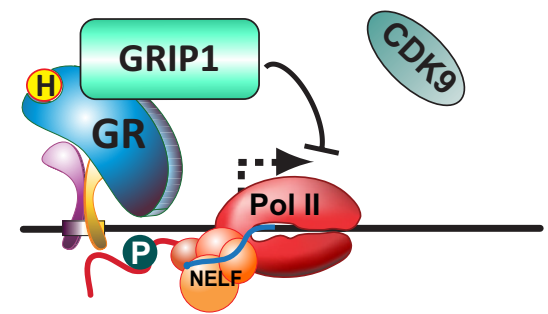

Elongation-controlled genes

B

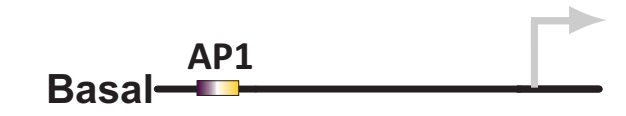

C

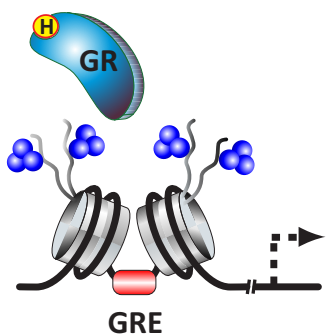

Induced
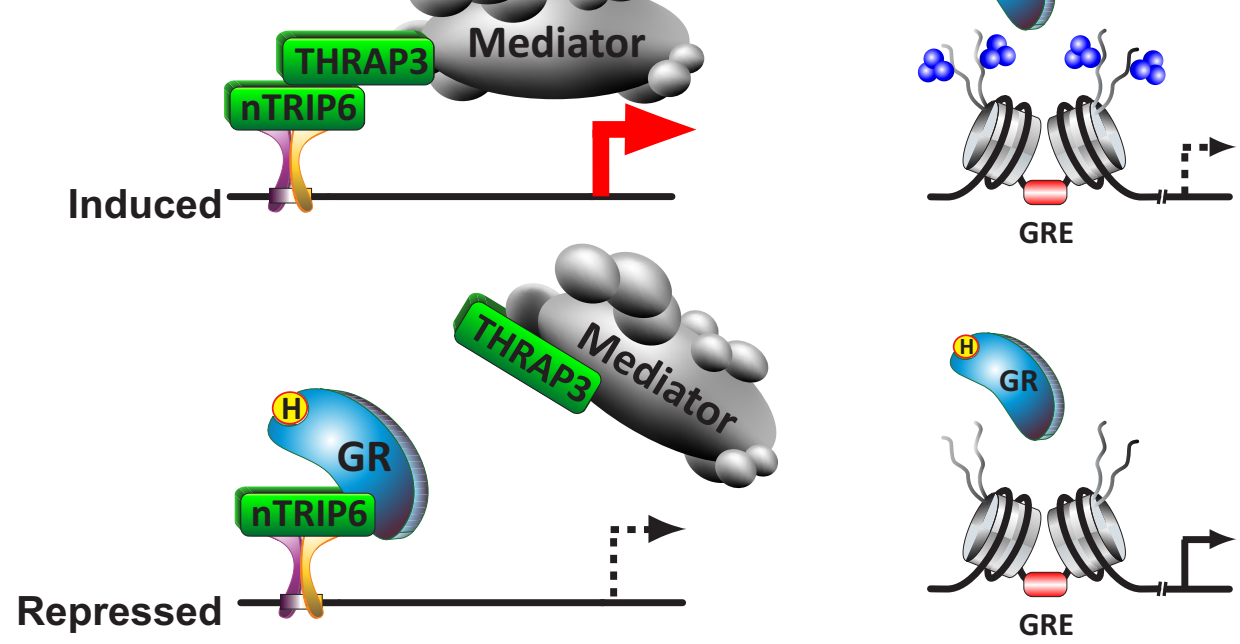
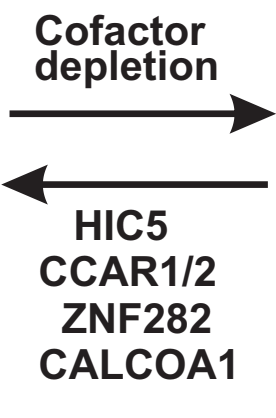

GRE
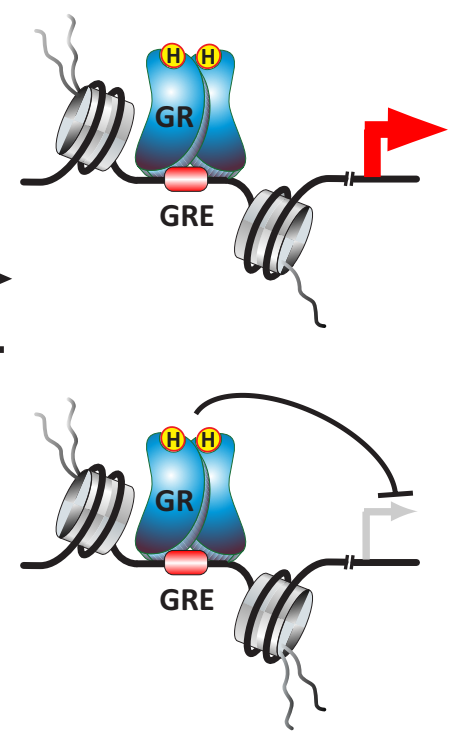
